\title{
Enfermedad de Ménière en adultos
}

\section{Meniere's Disease in Adults}

Pág. 96,105

Recibido: 04-05-2020

Aceptado: 21-06-2020

Dra. Sabrina Acosta Egea ${ }^{1}$

Dra. Marisabel Echeverría Miranda ${ }^{2}$

Dr. Esteban Salas Salas ${ }^{3}$

1,2. Médico General, San José, Costa Rica.

3. Médico General, Hospital Dr. Rafael Ángel Calderón Guardia.

\section{RESUMEN}

La enfermedad de Ménière es una patología crónica del oído interno que se caracteriza por episodios de vértigo espontáneo y recurrente, pérdida auditiva unilateral neurosensorial, tinnitus y plenitud ótica. Actualmente la causa de la enfermedad de Ménière es desconocida, se considera de origen multifactorial y el diagnóstico se basa en la presencia de vértigo episódico asociado a una pérdida auditiva neurosensorial. Las pruebas vestibulares miden el funcionamiento del oído interno, establecen un pronóstico y un tratamiento pero no son necesarias para realizar el diagnóstico. El manejo consiste en cambios en el estilo de vida, tratamiento médico y cirugía, todos estos con el fin de reducir la frecuencia y severidad de los síntomas y limitar la progresión de la pérdida auditiva.

\section{PALABRAS CLAVE}

Enfermedad de Ménière; Vértigo; Pérdida Auditiva

\section{ABSTRACT}

Meniere's Disease is a chronic illness of the inner ear characterized by episodic spontaneous and recurrent vertigo's, unilateral sensorineural hearing loss, tinnitus and aural pressure. Currently, the cause of Meniere's Disease is unknown, it is considered to be multifactorial and it's diagnosis is based on the presence of episodic vertigo associated to sensorineural hearing loss. Vestibular tests monitor the functionality of the inner ear, establish a prognosis and a treatment but are not necessary for diagnosis. Current management consists on life style changes, pharmacologic and surgical treatment. All of this in order to reduce the frequency and severity of symptoms and limit hearing loss progression. 


\section{KEY WORDS}

Meniere Disease; Vertigo; Hearing Loss

\section{INTRODUCCIÓN}

La enfermedad de Ménière se define como una enfermedad crónica del oído interno que se caracteriza por presentar episodios de vértigo espontáneo y recurrente, pérdida auditiva unilateral neurosensorial fluctuante, tinnitus y plenitud ótica $(1,2)$. Cuando este grupo de síntomas no se puede atribuir a una causa específica, se considera como idiopático y se le denomina enfermedad de Ménière (1).

Tiene una incidencia de $15-20$ casos en 100,000 habitantes (2) con una leve predominancia femenina (1). Su etiología es multifactorial, con el hidrops endolinfático como signo característico. Sin embargo, su relación con la enfermedad de Ménière es poco clara y en estudios recientes se sugiere que se necesitan factores adicionales para provocar la sintomatología que se presenta en esta enfermedad (3).

La enfermedad de Ménière se clasifica según los criterios de la Academia Americana de Otorrinolaringología y Cirugía de Cabeza y Cuello en definitivo y probable, y no es necesario detectar hidrops endolinfático para hacer dicho diagnóstico. Esta clasificación depende exclusivamente en el diagnóstico clínico, historia clínica y exámenes complementarios del oído interno (3). El objetivo del tratamiento en el síndrome de Ménière es mejorar la sintomatología durante los cuadros agudos, prevenir ataques recurrentes e impedir el daño progresivo de la función auditiva y vestibular del paciente (3).

Esta revisión pretende resaltar la cronicidad de la enfermedad y el difícil manejo que representa para los médicos, así como proporcionar una guía actualizada y resumida para el manejo clínico.

\section{METODO}

Se realizó una revisión bibliográfica donde se utilizaron artículos desde el 2015 hasta el 2020. Se seleccionaron de bases de datos de Elsevier, Pubmed, BMJ Journals, Scielo, Cochrane Library y Google Scholar. Se utilizaron palabras de búsqueda como "Enfermedad de Ménière," "manejo, " "clínica de Ménière" y "pronóstico." Se tomaron en cuenta solamente aquellos artículos cuyas fuentes tuvieran validez, fueran realizados por médicos especialistas en el área de interés o de hospitales reconocidos. Se seleccionaron un total de 17 artículos tanto de revisión como experimentales, todos en el idioma inglés.

\section{EPIDEMOLOGIA}

La enfermedad de Ménière tiene una aparición promedio entre los 40-50 años (3). La prevalencia aumenta con la edad y hay un pico a los 61-70 años (3). En niños dicha enfermedad es menos común con un porcentaje reportado entre 0.4$7 \%$ de todos los casos de enfermedad de Ménière y la mayoría son pacientes $>10$ años (3). Sin embargo, se han reportado casos de niños diagnosticados tan temprano como los 4 años de edad (3).

La enfermedad de Ménière tiene una incidencia de $15-20$ casos en 100,000 habitantes con un leve predominio hacia el sexo femenino (2). No se ha reportado diferencia entre una posible predominancia hacia el oído derecho o izquierdo, pero sí se ha visto que conforme aumenta la edad del paciente hay una mayor posibilidad de presentar una enfermedad de Ménière bilateral (2). Esta afectación bilateral 
se reporta en $25-40 \%$ de todos los casos y se asocia con un aumento de síntomas vestibulares y un mayor impacto negativo en la calidad de vida del paciente (2).

Se ha visto una alta prevalencia de migraña en pacientes con Enfermedad de Ménière y es más común en aquellos con antecedentes familiares de vértigo episódico (1). También se ha observado una relación genética de tipo autosómica dominante tanto en casos esporádicos como familiares $(1,4)$. Para que un caso se pueda describir como enfermedad familiar de Ménière, tiene que haber al menos un pariente de primer o segundo grado con criterios sugestivos de esta patología $(1,4)$. Se ha reportado enfermedad familiar de Ménière en $6-8 \%$ de casos esporádicos con múltiples genes alterados como FAM136A, DTNA, PRKCB, SEMA3D y DPT, lo cual sugiere una heterogeneidad genética $(1,4)$.

\section{ETIOLOGIA Y FISIOPATOLOGIA}

La causa de la enfermedad de Ménière es desconocida, ya que no se ha encontrado un único origen que explique los síntomas vestibulares y auditivos de esta enfermedad y la progresión que tiene (5).

Actualmente, lateoría principal de la fisiopatología de esta enfermedad se basa en la formación de un hidrops endolinfático a nivel del ducto coclear y el sáculo del oído interno $(1,5)$ (Figura 1)6. El hidrops endolinfático es la única anormalidad anatómica encontrada en los pacientes con esta enfermedad y se ha visto que el volumen de esta aumenta hasta un $200 \%$ en comparación con una persona sana $(1,5)$. La etiología para que se produzca dicha alteración en el oído interno se cree que es multifactorial, con una probable afectación a nivel auto-inmune, infecciosa, endocrina, alérgica, vascular, autonómica, genética, idiopática o dietética, que llegan a producir un desequilibrio homeostático $(1,5)$. Sin embargo, esta teoría tiene su limitación, ya que no todos los pacientes con enfermedad de Ménière presentan hidrops endolinfático y los que sí lo presentan, no todos tienen síntomas de la enfermedad $(1,5)$.

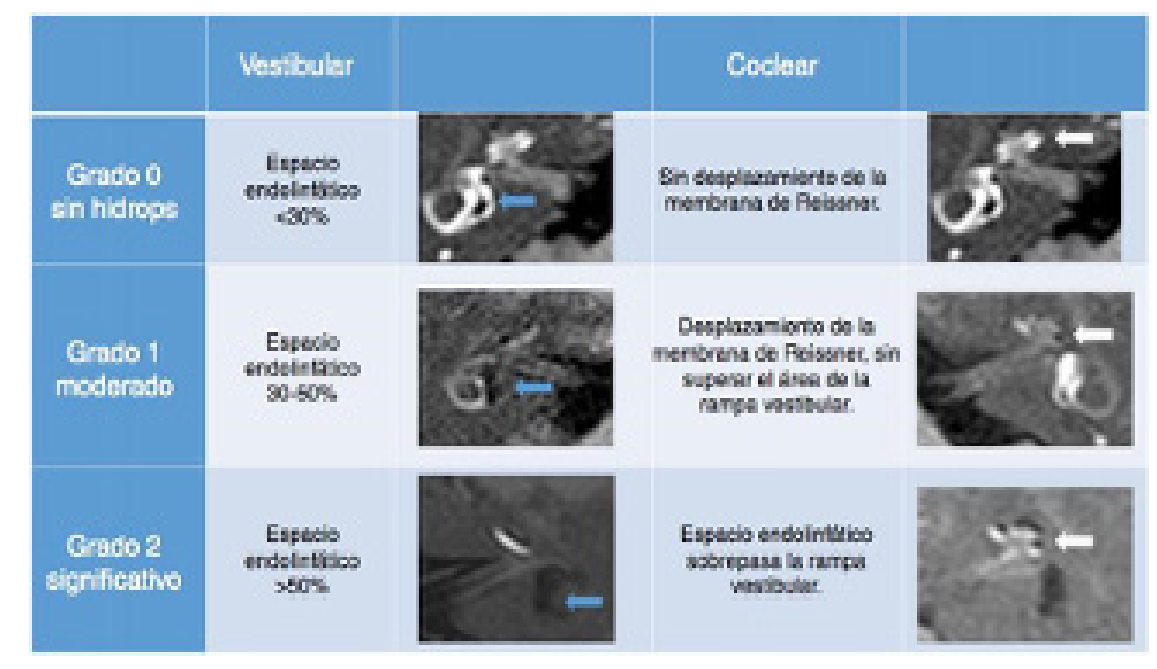

Figura 1. Esquema que clasifica los grados de hidrops endolinfático vestibular y coclear. Los hallazgos de describen como grado 0 (sin datos de hidrops), grado 1 (grado moderado de hidrops) y grado 2 (grado significativo de hiprops). Estas son imágenes mediante resonancia magnética de $3 \mathrm{~T}$ tras administración de gadolinio intratimpánico. 6

\section{CLINICA}

Los síntomas principales que se manifiestan en la enfermedad de Ménière son vértigo, pérdida auditiva y tinnitus $(1,7)$. Solo $40 \%$ de los pacientes presentan esta triada al inicio de la 
enfermedad $(1,7)$. Otros síntomas que también son reportados son: plenitud auricular que tiende a desaparecer conforme la enfermedad progresa, inestabilidad postural, náusea, crisis de Tumarkin y problemas de marcha $(1,7)$.

El vértigo recurrente espontáneo es el síntoma principal, se presenta en $96.2 \%$ de los pacientes y tiende a acompañar con náuseas, vómitos y diaforesis (1). Los cuadros de vértigo generalmente son precedidos por síntomas de tinnitus, plenitud auricular y alteraciones auditivas en el oído afectado (1) y pueden durar varias horas, pero aunque éstos disminuyen, los pacientes pueden presentar inestabilidad por varios días (1). Hasta un $72 \%$ de los pacientes presentan lo que se conoce como crisis de Tumarkin, que son caídas súbitas sin un factor precipitante como vértigo, pérdida de conciencia u otros síntomas neurológicos (1).

En un $77 \%$ de los pacientes se asocia una pérdida auditiva con los ataques de vértigo $(1,11)$. En las fases iniciales de la enfermedad, dicha perdida auditiva es reversible al terminar la crisis de vértigo; sin embargo, conforme progresa la enfermedad la pérdida auditiva neurosensorial se hace permanente $(1,11)$. Existe una forma clínica de presentación de la enfermedad de Ménière llamada el síndrome de Lermoyez, que consiste en una mejoría de la audición alrededor de la crisis vertiginosas, para luego volver a descender cuando esta termina $(1,11)$. Para algunos pacientes el tinnitus es el primer síntoma con que empieza la enfermedad de Ménière $(1,11)$. Al inicio, en un $83 \%$ de los pacientes, este síntomas es intermitente y solo aparece durante las crisis $(1,11)$. Sin embargo, conforme las crisis se vuelven recurrentes, la pérdida auditiva y el tinnitus se vuelven persistentes $(1,11)$. Algunos pacientes afirman que previo a una crisis el tono o la intensidad del tinnitus cambia y esto les indica que va a comenzar el vértigo $(1,11)$. Este síntoma es uno de los que más afecta la vida diaria del paciente $(1,11)$. Se han asociado múltiples factores precipitantes a los ataques de la enfermedad de Meniérè como: el estrés, algunos alimentos, alergenos, cambios de presión barométrica, cambios hormonales y falta de sueño (1).

\section{DIAGNOSTICO}

El diagnóstico de la enfermedad de Ménière se basaenlos criterios dediagnósticoformulados por la Academia Americana de Otorrinolaringología y Cirugía de Cabeza y Cuello y el Comité de Clasificación de la Sociedad Bárány $(3,7,8)$. Esta clasificación divide la enfermedad de Ménière en dos categorías: definitiva y probable $(3,7,8)$.

La enfermedad definitiva, se basa en la observación de vértigo episódico, asociado a una pérdida auditiva neurosensorial de baja a mediana frecuencia y síntomas auriculares fluctuantes como tinnitus o plenitud auricular en el oído afectado $(3,7,8)$. Los síntomas de vértigo duran entre 20 min a 12 horas $(3,7,8)$. En la enfermedad probable, se observan síntomas vestibulares episódicos como vértigo o mareo, asociado con síntomas auriculares fluctuantes por un periodo de $20 \mathrm{~min}$ a 24 horas $(3,7,8)$. En esta clasificación no es necesario el hallazgo de hidrops endolinfático para diagnosticar la enfermedad de Ménière $(3,7,8)$.

Las pruebas vestibulares no son necesarias para el diagnostico de la enfermedad de Ménière ya que no presentan hallazgos específicos (9). Sin embargo, estas son importantes para medir el funcionamiento del oído interno y ayudan a establecer un pronóstico y un tratamiento más específico (9). 
La prueba calórica es el método más antiguo utilizado para evaluar la función del sistema vestibular $(1,9)$. Hiporeflexia vestibular unilateral se ha observado en $75 \%$ de los pacientes con enfermedad de Ménière unilateral $(1,9)$. También se puede ver una prueba normal en hasta un $50 \%$ de los pacientes $(1,9)$. Es importante destacar que no existe un hallazgo patognomónico en esta prueba para la enfermedad de Ménière $(1,9)$.

Una pérdida auditiva neurosensorial de baja a medianas frecuencias es el hallazgo más significativo en la enfermedad de Ménière, por lo tanto es importante realizar una audiometría para el diagnóstico de esta enfermedad (8). Las frecuencias bajas (250-500 dB) son las que frecuentemente se afectan en estadíos iniciales de la enfermedad (1). Conforme está progresa, se puede ver una alteración en todas las frecuencias y un aplanamiento del audiograma (1).

La tomografía axial computarizada ha demostrado en pacientes con enfermedad de Ménière un acueducto vestibular más corto y estrecho comparado a pacientes sanos (1). Esto se observa en el oído afectado y en el contralateral (1). La resonancia magnética con contraste de gadolinio se utiliza para visualizar un posible hidrops endolinfático en el oído afectado o para buscar posibles anormalidades en el cerebro, realzar el conducto semicircular o buscar posibles displasias $(7,10)$.

\section{DIAGNOSTICO DIFERENCIAL}

Algunos diagnósticos diferenciales de la enfermedad de Ménière que se deben considerar son: un ataque isquémico transitorio, migraña vestibular y otros trastornos vestibulares (11).

Un $70 \%$ de los pacientes con enfermedad de
Ménière presentan cefalea y $30 \%$ presentan una comorbilidad de migraña (1). Es difícil diferenciar entre una migraña vestibular y una enfermedad de Ménière en pacientes que presentan vértigo y migraña recurrente (1). Los síntomas auditivos unilaterales es el criterio clínico diferencial más importante entre estas dos patologías (1). Sin embargo, se ha observado que los síntomas auditivos en la migraña vestibular aumentan conforme la enfermedad progresa, con pérdida auditiva en un $38 \%$ de los pacientes en un periodo de 9 años (1).

Puede ser útil realizar una resonancia magnética para excluir la posibilidad de un schwannoma vestibular o un tumor del saco endolinfático (11).

\section{TRATAMIENTO}

La enfermedad de Ménière no cuenta con una cura; por lo tanto, el objetivo principal del tratamiento es reducir la frecuencia y severidad de los síntomas, sobre todo en las crisis de vértigo $(12,13)$. El tratamiento debe ser en primera instancia conservador y dirigirse al síntoma que tiene mayor afectación en la vida diaria del paciente $(12,13)$. Actualmente el tratamiento se divide en farmacológico, intratimpánico y quirúrgico $(12,13)$.

\section{1) TRATAMIENTO FARMACOLÓGICO}

Durante el Congreso de la Federación Internacional de Sociedades de Otorrinolaringología (IFOS) en el 2017, se creó un algoritmo del tratamiento para la Enfermedad de Ménière con base en el conocimiento de seis especialistas de diferentes países $(13,14,15)$. En este algoritmo, se recomienda como primera línea de tratamiento hacer un cambio en el estilo de vida del paciente, mejorando su patrón de sueño, disminuyendo el estrés, evitando la

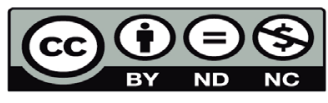


cafeína, alcohol, tabaco y comenzando una dieta baja en sodio $(13,14,15)$. La restricción de sal se recomienda para reducir el liquido del oído interno $(13,14,15)$. Sin embargo, realizar estos cambios en la dieta del paciente tienen poca evidencia de ser beneficiosos, por lo tanto, su uso es controversial $(13,14,15)$. Algunos autores piensan que al comenzar con el tratamiento conservador, esto retrasa el uso de un medicamento más efectivo que disminuya la progresión de la enfermedad $(13,14,15)$. Por representar esta medida de control dietético un bajo costo para el paciente, muchos profesionales lo utilizan en primera instancia $(13,14,15)$.

Añadido a los cambios en el estilo de vida, se debe considerar la rehabilitación vestibular y la psicoterapia cuando el paciente se encuentra en los periodos libres de crisis (13). La psicoterapia en especial se ha visto que genera un mejoría significativa hacia el deterioro funcional y los síntomas de náuseas que puede provocar esta enfermedad (13).

El tratamiento de segunda línea son los diuréticos $(12,15)$. Estos se comienzan cuando los síntomas de la enfermedad persisten a pesar de los cambios en el estilo de vida $(12,15)$. Los fármacos más utilizados son la hidroclorotiazida, acetazolamida y clortalidona $(12,15)$. Su función principal es disminuir las crisis de vértigo y otros síntomas de la enfermedad al reducir el volumen, presión o producción del líquido endolinfático $(12,15)$. Si el vértigo mejora al utilizar diuréticos, se puede pensar en quitar el tratamiento después de tres meses $(12,15)$. Se deben tener en cuenta las contraindicaciones y efectos secundarios de estos medicamentos antes de prescribirlos $(12,15)$.

El medicamento betahistina, antivertiginoso que se utiliza en Europa, Japón y Australia como otra línea de tratamiento, ya que puede actuar sobre el saco endolinfático y tiene pocos efectos secundarios $(16,17)$. Sin embargo, hay poca evidencia al respecto y no está aprobado por la FDA en Estados Unidos $(16,17)$.

\section{2) TRATAMIENTO POR VÍA INTRATIMPÁNICA}

Un $80 \%$ de los pacientes con enfermedad de Ménière mejoran sus síntomas con el tratamiento farmacológico (18). El uso de dexametasona, gentamicina 0

metilprednisolona por vía intratimpánica se ha vuelto cada vez más popular para tratar a aquellos pacientes que persisten con crisis de vértigo a pesar de mantener un cuidado preciso de la ingesta de sal (18). La dexametasona se utiliza con más frecuencia que la metilprednisolona y su dosis difiere con los autores, pero se ha visto mejoría con inyecciones de $4 \mathrm{mg} / \mathrm{mL}$ por cinco días consecutivos (18).

La gentamicina se utiliza ya que afecta selectivamente el sistema vestibular $(2,13)$. Actualmente se cree que es el tratamiento no quirúrgico más efectivo contra el vértigo $(2,13)$. Sin embargo, hay controversia sobre esta técnica, ya que puede provocar un leve deterioro auditivo $(2,13)$. Por lo tanto, se recomienda usar en pacientes que ya presentan deterioro auditivo significativo y que tengan un sistema vestibular contralateral preservado $(2,13)$.

Se ha observado que el tratamiento intratimpánico mejora significativamente la severidad y frecuencia de los vértigos en un periodo de 24 meses después de comenzar el tratamiento $(13,18)$. Hay una mejor respuesta en los pacientes con enfermedad de Ménière de etiología inflamatoria o autoinmunitaria, provocando una reducción significativa en los síntomas de vértigo y pérdida auditiva $(13,18)$. 
Sin embargo, la efectividad del esteroide tiende a ser temporal $(13,18)$. Aun así, su uso mejora las crisis de vértigo en un $25-50 \%$ de los pacientes $(13,18)$. En algunos pacientes el ciclo de tratamiento con esteroides se debe repetir después de la primera dosis, pero la respuesta mejora con cada inyección que se coloca $(13,18)$. El uso de esteroides es efectivo como tratamiento para las crisis de Tumarkin, tras uno o dos ciclos de este medicamento se han abolido dichas crisis por completo $(13,18)$.

\section{3) TRATAMIENTO QUIRÚRGICO}

Las técnicas quirúrgicas se utilizan en aquellos pacientes donde los tratamientos anteriores no han sido efectivos $(2,13)$. En los últimos años su uso ha disminuido por la eficacia del tratamiento intratimpánico $(2,13)$. El tratamiento quirúrgico se puede dividir en dos grupos: las técnicas conservadoras (cirugía de saco endolinfático y neurectomía vestibular) y destructivos (laberintectomía) $(2,13)$.

La descompresión del saco endolinfático es la técnica quirúrgica más utilizada en aquellos pacientes con función auditiva conservada y jóvenes, pero su eficacia es controversial $(2,13)$. Esta cirugía consiste en realizar una incisión en la pared lateral del lumen del saco para descomprimir el hidrops endolinfático $(2,13)$. Se han observado mejores resultados en esta cirugía al colocar altas dosis de esteroides cuando se abre el saco endolinfático $(2,13)$. La meta de esta técnica quirúrgica es mejorar las crisis de vértigo y preservar la función auditiva $(2,13)$.

La neurectomía vestibular es la técnica quirúrgica con mejores resultados en cuanto a la supresión de las crisis de vértigo y sus recurrencias (2). Tras esta cirugía, 90\% de los pacientes presentan una completa abolición de este síntoma y $92 \%$ presentan una preservación de la audición de >-10 Db (2). Esta cirugía se realiza en conjunto con neurocirugía y consiste en una craneotomía donde se secciona el nervio vestibular y se preserva el nervio coclear (2).

La técnica quirúrgica destructiva es la laberintectomía $(13,17)$. Esta se utiliza cada vez menos ya que destruye lo que queda de la función auditiva del paciente y su eficacia es muy parecida a la neurectomía vestibular $(13,17)$. Se realiza en pacientes que ya presentan alteraciones auditivas significativas, con un vértigo incapacitante, con crisis de Tumarkin y con enfermedad unilateral $(13,17)$. Se recomienda colocar un implante coclear tras realizar esta cirugía para tratar de rehabilitar la audición $(13,17)$.

Conforme la enfermedad de Ménière progresa, está tiende a volverse bilateral $(13,17)$. Este tipo de enfermedad se debe tratar de forma conservadora para prevenir una sordera bilateral $(13,17)$. Se tiende a utilizar la terapia intratimpánica con esteroides $(13,17)$. Si se debe hacer una técnica quirúrgica se debe realizar una descompresión del saco endolinfático a pesar de su controversia $(13,17)$.

\section{PRONOSTICO}

El curso de la enfermedad de Ménière es variable (1). Sin tratamiento, un $57 \%$ de los pacientes presentan control completo del vértigo en 2 años y $71 \%$ en 8.3 años (1). Usualmente, conforme la enfermedad progresa, las crisis de vértigo son menos frecuentes, la enfermedad se tiende a volver bilateral y el tinnitus y la pérdida auditiva se vuelven permanentes (1). Se creó una escala por la Academia Americana de Otorrinolaringología y Cirugía de Cabeza y Cuello donde se puede monitorizar el impacto

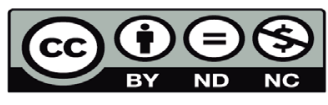


de la enfermedad de Ménière sobre la vida diaria de los pacientes (1). Se utiliza una escala de 6 puntos donde se evalúa los efectos del vértigo episódico en la vida diaria de los pacientes (Tabla 1$) 1$.

Con respecto a su estado actual de funcionamiento, no solo durante los ataques, marque el que mejor se aplique:

1) Mi mareo no tiene ningún efecto en mis actividades

2) Cuando estoy mareado, tengo que parar lo que estoy haciendo por un tiempo, pero pronto pasa y puedo reanudar las actividades. Yo sigo trabajando, conduciendo y participando en cualquier actividad que elija sin restricción alguna. No he cambiado ningún plan 0 actividad para acomodar mi mareo.

3) Cuando estoy mareado, tengo que parar lo que estoy haciendo por un tiempo, pero pasa y puedo reanudar las actividades. Yo sigo trabajando, conduciendo y participando en la mayoría de las actividades que elijo, pero he tenido que cambiar algunos planes $y$ hacer algunos acomodos para mi mareo.

4) Puedo trabajar, conducir, viajar y cuidar a una familia o participar en la mayoría de las actividades esenciales, pero dedo ejercer un gran esfuerzo para hacerlo. Debo hacer ajustes constantemente en mis actividades y dirigir mis energías. Apenas lo estoy logrando.

5) No puedo trabajar, conducir o cuidar a una familia. No puedo realizar la mayoría de las actividades que antes hacia. Incluso las actividades esenciales deben ser limitadas. Soy discapacitado.

6) He estado discapacitado durante 1 año o más y/o recibo compensación (dinero) debido a mi mareo o problema de balance.

\section{CONCLUSION}

La enfermedad de Ménière es una patología crónica del oído interno con síntomas de vértigo, pérdida auditiva neurosensorial y tinnitus. Esta enfermedad es de gran importancia tanto en medicina general como en otorrinolaringología por su afectación significativa en la vida diaria de los pacientes. Es importante que los médicos tengan conocimiento de este padecimiento para realizar un adecuado diagnóstico y abordaje. El diagnóstico de esta enfermedad se basa en criterios clínicos pero es importante realizar una audiometría y pruebas vestibulares para ayudar a establecer un pronóstico y un tratamiento más específico. Ya que la enfermedad de Ménière no tiene una etiología clara, hallar un tratamiento efectivo es complejo. Sin embargo, actualmente los tratamientos con los que se cuentan para esta enfermedad son: cambios en el estilo de vida, medicamentos orales tales como diuréticos, antivertiginosos y esteroides, tratamientos intratimpánicos o técnicas quirúrgicas. Con el progreso de la enfermedad, las crisis de vértigo tienden a disminuir pero el paciente presenta una pérdida auditiva y tinnitus persistente que en muchos casos afectan significativamente la vida diaria del paciente. Se necesitan estudios a nivel de Centro América que comparen los tratamiento utilizados para esta enfermedad y su efectividad ya que se encontró pocas referencias sobre el manejo en esta región.

\section{CONFLICTOS DE INTERES}

Los autores declaran que no existen conflictos de intereses ni beneficios económicos al realizar este artículo. 


\section{REFERENCIAS}

1. Espinosa-Sanchez JM, Lopez-Escamez JA. Menière's disease. Handb. Clin. Neurol. 2016 Jan 1 (Vol. 137, pp. 257-277). Elsevier. http://dx.doi.org/10.1016/B978-0-444-63437-5.00019-4

2. Kitahara T. Evidence of surgical treatments for intractable Meniere's disease. Auris Nasus Larynx. 2018 Jun 1;45(3):393-8. http://dx.doi.org/10.1016/j.anl.2017.07.016

3. Nakashima T, Pyykkö I, Arroll MA, Casselbrant ML, Foster CA, Manzoor NF, Megerian CA, Naganawa S, Young YH. Meniere's disease. Nat.Rev. 2016 May 12;2(1):1-8. http://dx.doi. org/10.1038/nrdp.2016.28

4. Gallego-Martinez A, Lopez-Escamez JA. Genetic architecture of Meniere's disease. Heares. 2019 Dec 13:107872. https://doi.org/10.1016/j.heares.2019.107872

5. Mirza S, Gokhale S. Pathophysiology of Meniere's disease. IntechOpen. 2017 Oct 4;1:53-67. http://dx.doi.org/10.5772/66388

6. Gómez MT, Duro DL, Álvarez BB, García-Berrocal JR. Diagnóstico del hidrops endolinfático mediante resonancia magnética de $3 \mathrm{~T}$ tras administración degadolinio intratimpánico. Radiología. 2017 Mar 1;59(2):159-65. http://dx.doi.org/10.1016/j.rx.2016.10.006

7. Gürkov R, Pyykö I, Zou J, Kentala E. What is Meniere's disease? A contemporary re-evaluation of endolymphatic hydrops. J Neurol. 2016 Apr 1;263(1):71-81. https://doi. org/10.1007/s00415-015-7930-1

8. Magnan J, Özgirgin ON, Trabalzini F, Lacour M, Escamez AL, Magnusson M, Güneri EA, Guyot JP, Nuti D, Mandalà M. European position statement on diagnosis, and treatment of Meniere's disease. J Int Adv Otol. 2018; Aug;14(2):317. http://dx.doi.org/10.5152/ iao.2018.140818

9. de Rezende Oliveira LN, de Andrada Oliveira CL, de Carvalho Lopes K, Ganança FF. Diagnostic assessment of patients with Meniere's disease through caloric testing and the video-head-impulse test. Braz J Otorhinolaryngol. 2019 Nov 22. https://doi.org/10.1016/j. bjorl.2019.10.008

10. Attyé A, Eliezer M, Galloux A, Pietras J, Tropres I, Schmerber S, Dumas G, Krainik A. Endolymphatic hydrops imaging: Differential diagnosis in patients with Meniere disease symptoms. Diagn Interv Imag. 2017 Oct 1;98(10):699-706. http://dx.doi.org/10.1016/j. diii.2017.06.002

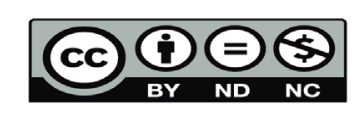


11. Lopez-Escamez JA, Carey J, Chung WH, Goebel JA, Magnusson M, Mandalà M, NewmanToker DE, Strupp M, Suzuki M, Trabalzini F, Bisdorff A. Diagnostic criteria for Menière's disease. J Vestibul Res-Equil. 2015 Jan 1;25(1):1-7. http://dx.doi.org/10.3233/VES-150549

12. Molnár A, Maihoub S, Tamás L, Szirmai Á. Conservative Treatment Possibilities of Ménière Disease, Involving Vertigo Diaries. Ear Nose Throat J. 2019 Oct 16:0145561319881838. http:// dx.doi.org/10.1177/0145561319881838

13. Nevoux J, Barbara M, Dornhoffer J, Gibson W, Kitahara T, Darrouzet V. International consensus (ICON) on treatment of Ménière's disease. Eur Ann Otorhinolaryngol Head Neck Dis. 2018 Feb 1;135(1):S29-32. https://doi.org/10.1016/j.anorl.2017.12.006

14. Hussain K, Murdin L, Schilder AG. Restriction of salt, caffeine and alcohol intake for the treatment of Ménière's disease or syndrome. Cochrane Database Syst. Rev. 2018(12). https:// doi.org/10.1002/14651858.CD012173.pub2

15. Stern Shavit S, Lalwani AK. Are diuretics useful in the treatment of meniere disease?. Laryngoscope. 2019 Oct;129(10):2206-7. http://doi.org/ 10.1002/lary.28040

16. Harcourt J, Cosentino S. Betahistine for Meniere's disease. Br. Med. J. 2016 Jan; 352:i46. https://doi.org/10.1136/bmj.i46

17. Nevoux J, Franco-Vidal V, Bouccara D, Parietti-Winkler C, Uziel A, Chays A, Dubernard X, Couloigner V, Darrouzet V, Mom T, de la SFORL GD. Diagnostic andtherapeutic strategy in Menière's disease. Guidelines of the French Otorhinolaryngology-Head and Neck Surgery Society (SFORL). Eur Ann

Otorhinolaryngol Head Neck Dis. 2017 Dec 1;134(6):441-4. https://doi.org/10.1016/j. anorl.2016.12.003

18. Patel M. Intratympanic corticosteroids in Meniere's disease: a mini-review. J. Otol. 2017 Sep 1;12(3):117-24. http://dx.doi.org/10.1016/j.joto.2017.06.002 